\title{
Linearization from Complex Lie Point Transformations
}

\author{
Sajid Ali, ${ }^{1}$ M. Safdar, ${ }^{2}$ and Asghar Qadir ${ }^{3}$ \\ ${ }^{1}$ School of Electrical Engineering and Computer Science, National University of Sciences and Technology, Campus H-12, \\ Islamabad 44000, Pakistan \\ ${ }^{2}$ School of Mechanical and Manufacturing Engineering, National University of Sciences and Technology, Campus H-12, \\ Islamabad 44000, Pakistan \\ ${ }^{3}$ School of Natural Sciences, National University of Sciences and Technology, Campus H-12, Islamabad 44000, Pakistan \\ Correspondence should be addressed to Sajid Ali; sajid_ali@mail.com
}

Received 19 August 2014; Revised 21 October 2014; Accepted 28 October 2014; Published 19 November 2014

Academic Editor: Peter G. L. Leach

Copyright (C) 2014 Sajid Ali et al. This is an open access article distributed under the Creative Commons Attribution License, which permits unrestricted use, distribution, and reproduction in any medium, provided the original work is properly cited.

Complex Lie point transformations are used to linearize a class of systems of second order ordinary differential equations (ODEs) which have Lie algebras of maximum dimension $d$, with $d \leq 4$. We identify such a class by employing complex structure on the manifold that defines the geometry of differential equations. Furthermore we provide a geometrical construction of the procedure adopted that provides an analogue in $\mathbb{R}^{3}$ of the linearizability criteria in $\mathbb{R}^{2}$.

\section{Introduction}

One method of solving a nonlinear ODE or a system of such equations is to reduce it to linear form, which is called linearization, by invertible transformations of the independent and dependent variables (point transformations). There are many ways to convert to linear form like Lie point transformations, contact transformations, nonlocal transformations, and so forth, but for convenience we will use the term "linearization" to signify the Lie point transformations only and qualify the term if we mean any other transformation. Lie presented the most general form of a scalar second order linearizable ODE by considering arbitrary point transformations (see, e.g., $[1,2]$ and the references therein). Over the past few years there has been a rapidly growing interest in studying linearization of higherorder ODEs and systems of these equations. The simplest system $\left(f_{1}^{\prime \prime}=0, f_{2}^{\prime \prime}=0\right)$ is a unique system that admits a maximal algebra of dimension $15=|\mathrm{sl}(4, \mathbb{R})|$, which was proved in [3], using Cartan's equivalence method, after refining the result in [4]. A geometric approach is used to obtain linearization criteria for systems of two second order cubically semilinear ODEs obtainable by projecting down a system of three geodesic equations $[5,6]$. Indeed, utilizing arbitrary point transformations, general forms of linearizable systems and corresponding linearization criteria were studied in [7-9]. The use of generalized Sundman transformations in the linearization problem is studied in [10]. The construction of linearizing transformations from the first integrals of twodimensional systems is carried out in $[11,12]$.

It is well known [13] that to be linearizable by Lie point transformation a system of two second order ODEs can have an algebra of maximum dimensions, 5, 6, 7, 8, or 15. The systems which do not have maximal algebras are clearly nonlinearizable in the sense that there exist no real Lie point transformation which can be used to linearize them. This paper addresses the problem of linearizability of such a class of nonlinearizable systems by complex Lie point transformations.

Recently we studied a special class of two-dimensional linearizable systems that corresponds to complex linearizable scalar ODEs $[14,15]$. (The characterization for the correspondence will be explained in the next section.) The linearizing transformations to map nonlinear systems to linear forms are provided by the complex fibre-preserving transformations:

$$
\mathscr{L}_{1}:(x, u(x)) \longrightarrow(\chi(x), U(x, u)),
$$

where $u(x)$, is an analytic complex function of a real variable $x$. The method was successfully applied to generate classes of 
systems with maximum dimensions of their algebras 6,7, and 15 , where the linearizing transformations were obtained by the process of "realification" of the above transformations (1). In this paper we investigate a nonlinearizable class of systems of two ODEs that can be obtained from a linearizable scalar complex equation, but the complex linearizing transformations in this case are different from those given above; that is,

$$
\mathscr{L}_{2}:(x, u(x)) \longrightarrow(\chi(x, u), U(x, u))
$$

which is a complex Lie point transformation and contains (1) as its special case. Notice that $\mathscr{L}_{2}$ cannot be used to obtain the linearizing transformations for the corresponding systems. The reason is that the transformed independent variable is complex which gives two independent variables when it splits into the real and imaginary parts while the concerned systems are of ODEs rather than PDEs. The standard techniques of linearization $[8,9,13]$ cannot be applied to such systems as they have symmetry algebra of maximum dimension $d \leq 4$. However we prove that linearization can still be achieved for such systems in the complex plane and the solutions can also be obtained explicitly. Most surprisingly, we find that there can be systems that have no Lie point symmetries but can be solved by this procedure of converting to a complex scalar ODE and linearizing the scalar equation to write down the solution in the complex domain and thence obtain the solution of the system. An example of such a system is also given. We call this procedure for linearizing systems of two second order ODEs from complex point transformations with less than five symmetries complex linearization.

The procedure of constructing a pair of real functions of two variables from a single complex function of a complex variable, leading to a system of PDEs, entails the use of the Cauchy-Riemann (CR) equations in a transparent way. However, the role of these equations for a system of ODEs is far from clear as the CR-equations require two independent variables. Here we give a simple explanation of this role using geometry, for the free particle ODE which presents an elegant framework of viewing the straight line (which is maximally symmetric and invariant under the full group $\operatorname{SL}(3, \mathbb{R})$ ) in a higher dimensional space which is only possible if we put on complex glasses. The CR-equations are shown to play an essential part in establishing the correspondence between solutions of the base complex linearizable or integrable ODEs and emerging systems.

The outline of the paper is as follows. The criteria for the correspondence of systems with complex linearizable equations are given in the second section. The third section is on the CR-equations associated with systems of two second order ODEs and exploring their role in establishing correspondence of solutions of systems and base scalar ODEs. The subsequent section contains application of complex linearization procedure on systems which correspond to linearizable complex base ODEs. The last section is devoted to the conclusion and discussion.

\section{Complexification of Systems of ODEs and Classification}

We first explain the basic formalism of complex linearizability by taking a general system of two second order ODEs:

$$
\begin{aligned}
& f_{1}^{\prime \prime}=\omega_{1}\left(x, f_{1}, f_{2}, f_{1}^{\prime}, f_{2}^{\prime}\right), \\
& f_{2}^{\prime \prime}=\omega_{2}\left(x, f_{1}, f_{2}, f_{1}^{\prime}, f_{2}^{\prime}\right),
\end{aligned}
$$

which may be regarded as a surface $S$ in a $2(3)+1=7$ dimensional space whose components comprise the independent and dependent variables along with their derivatives. Hence a solution of system (3) is an integral curve on surface $S$. We now introduce a complex structure $J_{\mathbb{C}}: \mathbb{R}^{6} \rightarrow \mathbb{C}^{3}$, on the 6 -dimensional subspace of $S$ by assuming $f_{1}(x)+i f_{2}(x)=$ $u(x)$, where all first order and second order derivatives of the real functions $f_{1}, f_{2}$ of a real variable $x$ are determined with $u^{\prime}$ and $u^{\prime \prime}$, respectively. Therefore our solution curve

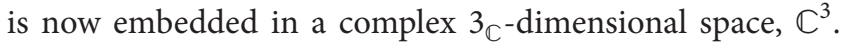
If we regard $x$ as the dimension of time then this can be viewed as the propagation of our solution curve in time in a complex space $\mathbb{C}^{3}$. This identification gives deeper insights into the symmetry analysis which we will see as we proceed to the subsequent sections. This yields a class of those twodimensional systems (3) which can be projected to a scalar second order complex equation:

$$
u^{\prime \prime}=\omega\left(x, u, u^{\prime}\right)
$$

where $\omega(x, u)=\omega_{1}(x, u)+i \omega_{2}(x, u)$, in a $3_{\mathbb{C}}+1=$ 4 -dimensional partially complex space $S_{\mathbb{C}}$, which is comprised of a $3_{\mathbb{C}}$-complex dimensional subspace and a onedimensional subspace that correspond to independent variable $x$ which is not complex. The basic criterion to identify such systems is that both $\omega_{1}$ and $\omega_{2}$ in (3) satisfy the CRequations:

$$
\begin{array}{ll}
\omega_{1, f_{1}}=\omega_{2, f_{2}}, & \omega_{1, f_{2}}=-\omega_{2, f_{1}}, \\
\omega_{1, f_{1}^{\prime}}=\omega_{2, f_{2}^{\prime}}, & \omega_{1, f_{2}^{\prime}}=-\omega_{2, f_{1}^{\prime}},
\end{array}
$$

namely, both functions are analytic of their arguments. This line of approach has been followed in $[14,15]$ to characterize systems of ODEs that emerge from linearizable complex equations. Several nontrivial and interesting results are obtained for two-dimensional systems despite "trivial" identification of systems (3) from scalar equations (4).

A two-dimensional system of ODEs

$$
\begin{aligned}
f_{1}^{\prime \prime}= & A_{1} f_{1}^{\prime 3}-3 A_{2} f_{1}^{\prime 2} f_{2}^{\prime}-3 A_{1} f_{1}^{\prime} f_{2}^{\prime 2}+A_{2} f_{2}^{\prime 3} \\
& +B_{1} f_{1}^{\prime 2}-2 B_{2} f_{1}^{\prime} f_{2}^{\prime}-B_{1} f_{2}^{\prime 2}+C_{1} f_{1}^{\prime}-C_{2} f_{2}^{\prime}+D_{1}, \\
f_{2}^{\prime \prime}= & A_{2} f_{1}^{\prime 3}+3 A_{1} f_{1}^{\prime 2} f_{2}^{\prime}-3 A_{2} f_{1}^{\prime} f_{2}^{\prime 2}-A_{1} f_{2}^{\prime 3} \\
& +B_{2} f_{1}^{\prime 2}+2 B_{1} f_{1}^{\prime} f_{2}^{\prime}-B_{2} f_{2}^{\prime 2}+C_{2} f_{1}^{\prime}+C_{1} f_{2}^{\prime}+D_{2},
\end{aligned}
$$

where $A_{j}, B_{j}, C_{j}, D_{j}(j=1,2)$ are functions of $x$ and $f_{j}$, is a candidate of complex linearization if and only if its 
coefficients satisfy a set of four constraint equations given in [15]. It was shown that the system (6) corresponds to a cubically semilinear complex scalar equation of the form

$$
\begin{aligned}
u^{\prime \prime}+ & E_{3}(x, u) u^{\prime 3}+E_{2}(x, u) u^{\prime 2}+E_{1}(x, u) u^{\prime} \\
& +E_{0}(x, u)=0
\end{aligned}
$$

where $E_{k},(k=0,1,2,3)$ are complex functions of $x$ and $u$, which according to Lie is linearizable using complex point transformations provided the coefficients satisfy linearization criteria given in [2]. Therefore system (6) is complex linearizable if and only if the associated base equation (7) can be mapped to $U^{\prime \prime}=0$, using a complex Lie point transformation (2). It may be pointed out that there is only one candidate of complex linear equation which is $U^{\prime \prime}=0$, unlike systems of two linear ODEs which have five candidates corresponding to their algebras $5,6,7,8$, or 15 of maximum dimensions. Therefore to be linearizable by real Lie point transformations system (6) can have one of these algebras.

The systems of the form (6) that correspond to a complex linear equation using fibre-preserving transformations (1) were studied in [14], where it was found that they are at most quadratic in the first derivatives and can have algebras of maximum dimensions 6,7 , or 15 . They can also be linearized using real Lie point transformations by standard techniques because the dimensions of their algebras coincide with the dimensions of linearizable systems. We denote this class of quadratically semilinear systems by $\Upsilon_{1}$. It turns out that if we take systems (6) with cubic dependence on the first derivatives they are no longer linearizable because the dimensions of their Lie algebras do not coincide with the dimensions of linearizable systems. Therefore we obtain a class $\Upsilon_{2}$, of systems of the form (6), with cubic nonlinearity and such that the maximum dimensions of their Lie algebras can be $4,3,2,1$, or 0 . Such systems are not linearizable but they correspond to a linearizable (complex) scalar second order ODE. Now all systems of the form (6) for which coefficients satisfy constraint equations [15] correspond to complex linear equation (7) under the complex point transformation (2); therefore all systems in $\Upsilon_{2}$ are also complex linearizable. Since the dimensions of Lie algebras of systems in classes $\Upsilon_{1}$ and $\Upsilon_{2}$ are not equal, therefore there do not exist any complex point transformations that map a system in $\Upsilon_{1}$ to a system in $\Upsilon_{2}$ and vice versa; therefore

$$
\Upsilon_{1} \cap \Upsilon_{2}=\emptyset
$$

In short, the class $\Upsilon_{1}$ contains those complex linearizable systems which are at most quadratically semilinear and they can be mapped to the optimal canonical form [14], whereas all complex linearizable systems with cubic nonlinearity are contained in $\Upsilon_{2}$. Below we summarize two classes arising from a complex linear equation in Table 1.

Note that the cases of Lie algebras of maximum dimensions 5 and 8 are not contained in $\Upsilon_{1} \cup \Upsilon_{2}$ but do give linearizable systems. For completeness we also discuss a class of those systems that can be solved from complex methods in which systems are solved due to their correspondence with complex solvable equation. It is given in the appendix.
TABLE 1: Classification of two-dimensional systems arising from linear complex equation.

\begin{tabular}{lcccc}
\hline Class & $\begin{array}{c}\text { Lie algebra } \\
\text { dimensions }\end{array}$ & Linearizable & $\begin{array}{c}\text { Complex } \\
\text { linearizable }\end{array}$ & Type \\
\hline$\Upsilon_{1}$ & $6,7,15$ & Yes & Yes & Quadratic \\
$\Upsilon_{2}$ & $0,1,2,3,4$ & No & Yes & Cubic \\
\hline
\end{tabular}

In order to construct the representative system for the class $\Upsilon_{2}$ with fewer symmetries yet is complex linearizable we focus on a class of cubically semilinear systems:

$$
\begin{aligned}
& f_{1}^{\prime \prime}=\beta f_{1}^{\prime 3}-3 \gamma f_{1}^{\prime 2} f_{2}^{\prime}-3 \beta f_{1}^{\prime} f_{2}^{\prime 2}+\gamma f_{2}^{\prime 3}, \\
& f_{2}^{\prime \prime}=\gamma f_{1}^{\prime 3}+3 \beta f_{1}^{\prime 2} f_{2}^{\prime}-3 \gamma f_{1}^{\prime} f_{2}^{\prime 2}-\beta f_{2}^{\prime 3},
\end{aligned}
$$

from (6), where $\beta=\beta\left(x, f_{1}, f_{2}\right)$ and $\gamma=\gamma\left(x, f_{1}, f_{2}\right)$. The complex linearizability criteria [15] are satisfied for these systems if and only if the coefficients satisfy

$$
\beta_{x x}=0, \quad \gamma_{x x}=0 ;
$$

that is, $\beta=b_{1} x+b_{2}, \gamma=c_{1} x+c_{2}$, therefore the system (9) is complex linearizable. It is easy to verify that the systems (9) have symmetry algebras of maximum dimensions less than 4 , provided all constants $b_{1}, b_{2}, c_{1}$, and $c_{2}$ do not vanish simultaneously. The system (9) satisfies (5) and thus can be projected to (7):

$$
u^{\prime \prime}+E_{3}(x, u) u^{\prime 3}=0
$$

with $E_{2}=0=E_{1}=E_{0}$, which can be linearized to $U^{\prime \prime}=0$, using a complex point transformation (2). Before proceeding to the applications and characterizing such systems we first describe the geometry of $U^{\prime \prime}=0$, under general complex Lie point transformations (2).

\section{Geometry of Complex Linearization}

We know that all linearizable scalar differential equations are equivalent to the free particle equations (see, e.g., $[1,2]$ ) whose solution is a straight line. The crucial step after ensuring complex linearizability is to obtain the transformations which help in the integration of the systems in $\Upsilon_{1}$ and $\Upsilon_{2}$. In [14], the complex fibre-preserving transformations (1) were used to map a system in class $\Upsilon_{1}$ into the free particle complex equation, $U^{\prime \prime}=0$, where prime denotes differentiation with respect to $\chi$. The real and imaginary parts of such a free particle equation yields a system

$$
F_{1}^{\prime \prime}=0, \quad F_{2}^{\prime \prime}=0,
$$

where $U=F_{1}+i F_{2}$; therefore the corresponding system can be mapped to system (12), where the complex transformations (1) were used to obtain the real linearizing transformations. It is noteworthy that the transformations in this case are (real,complex $) \rightarrow$ (real,complex). On the other hand a general complex point transformation is of the form

$$
\mathscr{L}:(x, u) \longrightarrow(\chi(x, u), U(x, u)),
$$


namely, (real, complex $) \rightarrow$ (complex, complex), in which the first argument $\chi$, can be a complex function thereby adds a superficial dimension. It makes a huge difference on linearizability of systems in $\Upsilon_{2}$. In particular, upon splitting it into the real and imaginary parts

$$
\chi(x, u)=\chi_{1}\left(x, f_{1}, f_{2}\right)+i \chi_{2}\left(x, f_{1}, f_{2}\right)
$$

and since the dependent function $U(\chi)$ is complex which yields two real functions $F_{1}$ and $F_{2}$, both of them are not only functions of $\chi_{1}$ but also of $\chi_{2}$; that is,

$$
U(\chi)=F_{1}\left(\chi_{1}, \chi_{2}\right)+i F_{2}\left(\chi_{1}, \chi_{2}\right) ;
$$

therefore the linearized scalar equation, $U^{\prime \prime}=0$, fails to produce the free particle system (12). Notwithstanding, the solution of a system in class $\Upsilon_{2}$ is extractable from the complex solution $U(x)$, upon its split. We now give a geometrical understanding of complex linearization associated with base equation $U^{\prime \prime}=0$. Since the prime denotes differentiation with respect to $\chi$ which upon using the chain rule yields

$$
\frac{\partial}{\partial \chi}=\frac{1}{2}\left(\frac{\partial}{\partial \chi_{1}}-i \frac{\partial}{\partial \chi_{2}}\right)
$$

and so $U^{\prime \prime}=0$ is a system of two partial differential equations

$$
\begin{aligned}
& F_{1_{\chi_{1} \chi_{1}}}-F_{1_{\chi_{2} \chi_{2}}}+2 F_{2_{\chi_{1} \chi_{2}}}=0, \\
& F_{\chi_{\chi_{1} \chi_{1}}}-F_{2_{\chi_{2} \chi_{2}}}-2 F_{1_{\chi_{1} \chi_{2}}}=0 .
\end{aligned}
$$

Now by definition a complex Lie point transformation is analytic thus $\mathscr{L}$ is analytic. Since the derivative $u^{\prime}$ transforms into a complex derivative $U^{\prime}$ which exists if and only if $U(\chi)$ is complex analytic and is preserved under $\mathscr{L}$ therefore

$$
F_{1_{\chi_{1}}}=F_{2_{\chi_{2}}}, \quad F_{1_{\chi_{2}}}=-F_{2_{\chi_{1}}},
$$

which are the CR equations. The solution of system (17) along with the condition (18) upon using invertible transformations (2) reveals solutions of the original system. Hence we have established the following result.

Theorem 1. All complex linearizable two-dimensional systems of ODEs in class $\Upsilon_{2}$ can be transformed into systems of PDEs (17) and (18) under the transformation (2).

We now develop some geometrical aspects of complex linearization. In order to do that we employ the original idea of Riemann that a complex mapping may be regarded as the dependence of one plane on another plane, unlike the dependence of a real function on a line. To do this we first obtain the solution of system (17)-(18) which is

$$
\begin{aligned}
& F_{1}\left(\chi_{1}, \chi_{2}\right)=c_{1} \chi_{1}+c_{2} \chi_{2}+c_{3}, \\
& F_{2}\left(\chi_{1}, \chi_{2}\right)=c_{1} \chi_{2}-c_{2} \chi_{1}+c_{4},
\end{aligned}
$$

where $c_{m},(m=1,2,3,4)$ are real arbitrary constants. These are two coordinate planes determined by $\chi_{1}$ and $\chi_{2}$ with normals

$$
\begin{gathered}
\mathbf{n}_{1}=\left[c_{1}, c_{2}\right] ; \\
\mathbf{n}_{2}=\left[c_{2},-c_{1}\right],
\end{gathered}
$$



FIGURE 1: The geometry of complex linearization. The straight line on the left is a solution of a linear second order real ODE. The concept naturally extends in the complex domain where a straight line arises in the intersection of two perpendicular planes determined by $F_{1}$ and $F_{2}$ which are linear functions of $\chi_{1}$ and $\chi_{2}$. The third axis is then naturally given by $\vec{n}_{1} \times \vec{n}_{2}$.

thus they intersect at right angles

$$
\mathbf{n}_{1} \cdot \mathbf{n}_{2}=0,
$$

resulting in a straight line at intersection. Thus the geometric linearizing criterion for scalar second order differential equations; namely, a straight line is extended to the intersection of two planes at right angle in the complex linearization of two-dimensional systems. Note that both $\chi_{1}$ and $\chi_{2}$ in (16) are functions of $\left(x, f_{1}, f_{2}\right)$. Therefore the role of $\chi_{2}$ can be regarded as slicing the three-dimensional space $\mathbb{R}^{3}=$ $\left\{\left(x, f_{1}, f_{2}\right)\right\}$ into two coordinate planes. Interestingly, the solution $\left(f_{1}, f_{2}\right)$ of the system under consideration is found by solving (19) with the use of $F_{1}$ and $F_{2}$ from $U(\chi)$ in (2). Hence we arrive at the following geometrical result.

Theorem 2. The necessary and sufficient condition for a twodimensional system (6) to be complex linearizable is that the two planes determined by (19) intersect at right angle resulting in a straight line which corresponds to scalar linear equations.

Figure 1 illustrates the geometry and presents an elegant description of complex linearization.

Illustrative Example. We consider an example of a physical system known as coupled modified Emden system [2]:

$$
\begin{aligned}
& f_{1}^{\prime \prime}=-3 f_{1} f_{1}^{\prime}+3 f_{2} f_{2}^{\prime}-f_{1}^{3}+3 f_{1} f_{2}^{2}, \\
& f_{2}^{\prime \prime}=-3 f_{2} f_{1}^{\prime}-3 f_{1} f_{2}^{\prime}+f_{2}^{3}-3 f_{1}^{2} f_{2},
\end{aligned}
$$

to explain briefly how the procedure works. This system has three symmetries $X_{1}, X_{2}$, and $X_{3}$, where

$$
\begin{gathered}
X_{1}=\frac{\partial}{\partial x}, \quad X_{2}=x \frac{\partial}{\partial x}-f_{1} \frac{\partial}{\partial f_{1}}-f_{2} \frac{\partial}{\partial f_{2}}, \\
X_{3}=x^{2} \frac{\partial}{\partial x}-2 x f_{1} \frac{\partial}{\partial f_{1}}-2 x f_{2} \frac{\partial}{\partial f_{2}},
\end{gathered}
$$


with Lie algebra $\left[X_{1}, X_{2}\right]=X_{1},\left[X_{1}, X_{3}\right]=2 X_{2}$, and $\left[X_{2}, X_{3}\right]=X_{3}$. The system (22) is solvable only by complex linearization. The complex point transformation

$$
\chi=x-\frac{1}{u}, \quad U=\frac{x^{2}}{2}-\frac{x}{u},
$$

is of the form (2), which does the trick to map the associated complex equation (4) of (22), into the complex free particle equation, whose solution after inverting the above transformations directly yields the solution of the system (22). In this case (17) and (18) yield

$$
\begin{aligned}
& F_{1}=a_{1} \chi_{1}-a_{2} \chi_{2}+b_{1}, \\
& F_{2}=a_{2} \chi_{1}+a_{1} \chi_{2}+b_{2},
\end{aligned}
$$

where

$$
\begin{array}{cc}
\chi_{1}=x-\frac{f_{1}}{f_{1}^{2}+f_{2}^{2}}, & \chi_{2}=\frac{f_{2}}{f_{1}^{2}+f_{2}^{2}}, \\
F_{1}=\frac{x^{2}}{2}-\frac{x f_{1}}{f_{1}^{2}+f_{2}^{2}}, & F_{2}=\frac{x f_{2}}{f_{1}^{2}+f_{2}^{2}},
\end{array}
$$

obtained from (24). Now by solving (25), for $f_{1}$ and $f_{2}$, and by invoking equations (26), we get the solution

$$
\begin{aligned}
f_{1}(x)= & \left(2 x^{3}-6 x^{2} a_{1}+4\left(a_{2}^{2}+a_{1}^{2}-b_{1}\right) x+4 a_{1} b_{1}+4 a_{2} b_{2}\right) \\
\times & \times\left(x^{4}-4 x^{3} a_{1}+4\left(\left(a_{2}^{2}+a_{1}^{2}-b_{1}\right) x^{2}\right.\right. \\
& \left.\left.+2\left(a_{2} b_{2}+a_{1} b_{1}\right) x+b_{1}^{2}+b_{2}^{2}\right)\right)^{-1}, \\
f_{2}(x)= & \left(\left(2 x^{2}+4 b_{1}\right) a_{2}+4 b_{2}\left(x-a_{1}\right)\right) \\
\times & \times\left(x^{4}-4 x^{3} a_{1}+4\left(\left(a_{2}^{2}+a_{1}^{2}-b_{1}\right) x^{2}\right.\right. \\
& \left.\left.+2\left(a_{2} b_{2}+a_{1} b_{1}\right) x+b_{1}^{2}+b_{2}^{2}\right)\right)^{-1},
\end{aligned}
$$

of system (22).

\section{Applications}

We now illustrate the theory with the aid of examples on class $\Upsilon_{2}$, that is, the complex linearizable systems with algebras of dimensions $d \leq 4$. It may be pointed out that systems which cannot be dealt via standard Lie symmetry approach are solvable via symmetry approach! We also highlight the procedure with which we obtained these systems systematically.

4.1. Solvable System of 4-Dimensional Algebra. Considering $\beta\left(x, f_{1}, f_{2}\right)=1$ and $\gamma\left(x, f_{1}, f_{2}\right)=0$, in (9) we obtain a coupled system

$$
\begin{aligned}
& f_{1}^{\prime \prime}-f_{1}^{\prime 3}+3 f_{1}^{\prime} f_{2}^{\prime 2}=0, \\
& f_{2}^{\prime \prime}-3 f_{1}^{\prime 2} f_{2}^{\prime}+f_{2}^{\prime 3}=0,
\end{aligned}
$$

which is complex linearizable and has only four symmetries:

$$
\begin{gathered}
X_{1}=\frac{\partial}{\partial x}, \quad X_{2}=\frac{\partial}{\partial f_{1}}, \quad X_{3}=\frac{\partial}{\partial f_{2}}, \\
X_{4}=2 x \frac{\partial}{\partial x}+f_{1} \frac{\partial}{\partial f_{1}}+f_{2} \frac{\partial}{\partial f_{2}},
\end{gathered}
$$

with Lie algebra

$$
\begin{array}{ccc}
{\left[X_{1}, X_{2}\right]=0,} & {\left[X_{1}, X_{3}\right]=0, \quad\left[X_{2}, X_{3}\right]=0,} \\
{\left[X_{1}, X_{4}\right]=2 X_{1}, \quad\left[X_{2}, X_{4}\right]=X_{2}, \quad\left[X_{3}, X_{4}\right]=X_{3} ;}
\end{array}
$$

therefore, it is not in one of the linearizable classes of twodimensional systems. Now in order to carry out integration of system (28), we linearize the corresponding equation

$$
u^{\prime \prime}-u^{\prime 3}=0,
$$

which has an 8-dimensional Lie algebra. This can be mapped to the linear equation

$$
U^{\prime \prime}+1=0,
$$

by inverting the role of the independent and dependent variables $\chi=u, U=x$. It has the solution $2 U=-\chi^{2}+a \chi+b$, where $a$ and $b$ are complex constants, which in terms of the original variables becomes $u(x)= \pm \sqrt{a-2 x}+b$, and yields the solution

$$
\begin{aligned}
& f_{1}(x)= \pm\left(\frac{a_{1}-2 x+\sqrt{\left(a_{1}-2 x\right)^{2}+a_{2}^{2}}}{2}\right)^{1 / 2}+b_{1}, \\
& f_{2}(x)= \pm\left(\frac{-a_{1}+2 x+\sqrt{\left(a_{1}-2 x\right)^{2}+a_{2}^{2}}}{2}\right)^{1 / 2}+b_{2},
\end{aligned}
$$

of (28). System (28) can be solved by real symmetry analysis because it has four symmetry generators. However, we now proceed to systems that are not solvable by real symmetry methods as they have fewer symmetry generators than four.

4.2. Solvable System of 3-Dimensional Algebra. It is easy to construct a system from (9) which has only three symmetries. For example, we observe that in (9) the functions $\beta$ and $\gamma$ can be at most linear functions of independent variable $x$. Hence we obtain a complex linearizable system

$$
\begin{aligned}
& f_{1}^{\prime \prime}-x f_{1}^{\prime 3}+3 x f_{1}^{\prime} f_{2}^{\prime 2}=0, \\
& f_{2}^{\prime \prime}-3 x f_{1}^{\prime 2} f_{2}^{\prime}+x f_{2}^{\prime 3}=0,
\end{aligned}
$$

by involving $x$ linearly in the coefficients to remove the $x$-translation. Thus we obtain the following 3-dimensional Abelian Lie algebra:

$$
X_{1}=x \frac{\partial}{\partial x}, \quad X_{2}=\frac{\partial}{\partial f_{1}}, \quad X_{3}=\frac{\partial}{\partial f_{2}} .
$$


We follow the same procedure as developed in the previous case and solve the corresponding nonlinear equation:

$$
u^{\prime \prime}-x u^{\prime 3}=0
$$

It is transformable to a linear form $U^{\prime \prime}=-U$, which after inverting the variables gives

$$
u(x)=\arctan \left(\frac{x}{\sqrt{a-x^{2}}}\right)+b .
$$

System (34) is not solvable by real symmetry methods but by complex linearization.

4.3. Solvable System of 2-Dimensional Algebras. Consider the system

$$
\begin{aligned}
& f_{1}^{\prime \prime}-f_{1} f_{1}^{\prime 3}+3 f_{2} f_{1}^{\prime 2} f_{2}^{\prime}+3 f_{1} f_{1}^{\prime} f_{2}^{\prime 2}-f_{2} f_{2}^{\prime 3}=0, \\
& f_{2}^{\prime \prime}-f_{2} f_{1}^{\prime 3}-3 f_{1} f_{1}^{\prime 2} f_{2}^{\prime}+3 f_{2} f_{1}^{\prime} f_{2}^{\prime 2}+f_{1} f_{2}^{\prime 3}=0,
\end{aligned}
$$

which has only two Lie symmetries

$$
X_{1}=\frac{\partial}{\partial x}, \quad X_{2}=3 x \frac{\partial}{\partial x}+f_{1} \frac{\partial}{\partial f_{1}}+f_{2} \frac{\partial}{\partial f_{2}} .
$$

The system (38) is solvable due to its correspondence with the complex scalar second order ODE:

$$
u^{\prime \prime}-u u^{\prime 3}=0
$$

which is linearizable, despite having only a two-dimensional algebra. Notice that even a scalar second order ODE requires at least two symmetries to be solvable, while here complex linearization helps to solve a system of two ODEs. In the subsequent cases we provide the solution for a system with only one symmetry generator, which is insufficient to solve even a scalar second order ODE. Nevertheless, we can go further!

4.4. Solvable System of 1-Dimensional Algebra. Consider $\beta\left(x, f_{1}, f_{2}\right)=x f_{1}$ and $\gamma\left(x, f_{1}, f_{2}\right)=x f_{2}$, in (9) we obtain

$$
\begin{aligned}
& f_{1}^{\prime \prime}-x f_{1} f_{1}^{\prime 3}+3 x f_{2} f_{1}^{\prime 2} f_{2}^{\prime}+3 x f_{1} f_{1}^{\prime} f_{2}^{\prime 2}-x f_{2} f_{2}^{\prime 3}=0 \\
& f_{2}^{\prime \prime}-x f_{2} f_{1}^{\prime 3}-3 x f_{1} f_{1}^{\prime 2} f_{2}^{\prime}+3 x f_{2} f_{1}^{\prime} f_{2}^{\prime 2}+x f_{1} f_{2}^{\prime 3}=0
\end{aligned}
$$

This system is nonlinearizable as it has only a scaling symmetry $X_{1}=x \partial_{x}$. The corresponding scalar second order complex ODE is

$$
u^{\prime \prime}-x u u^{\prime 3}=0
$$

which has an 8-dimensional algebra and linearizes to

$$
U^{\prime \prime}+\chi U=0
$$

which is the Airy equation whose solutions are Airy functions extended to the complex plane. The solution of the complex linearized equation for $U(x)$ is given by

$$
U(\chi)=c_{1} \operatorname{Ai}(-\chi)+c_{2} \operatorname{Bi}(-\chi),
$$

where $\operatorname{Ai}(-\chi)$ and $\operatorname{Bi}(-\chi)$, are the two Airy functions. Inverting (44), we obtain a solution of the associated nonlinear equation which implicitly provides a solution:

$$
\begin{aligned}
& \Re\left(c_{1} \operatorname{Ai}\left(-f_{1}-i f_{2}\right)+c_{2} \operatorname{Bi}\left(-f_{1}-i f_{2}\right)\right)=x, \\
& \Im\left(c_{1} \operatorname{Ai}\left(-f_{1}-i f_{2}\right)+c_{2} \operatorname{Bi}\left(-f_{1}-i f_{2}\right)\right)=0,
\end{aligned}
$$

where $\mathfrak{R}$ and $\mathfrak{I}$ are the real and imaginary parts of the arguments, for the system (41).

4.5. Solvable System of 0-Dimensional Algebra. We now take the quadratic freedom of $f_{1}$ and $f_{2}$, in $\beta$ and $\gamma$, to get rid of the remaining symmetry in the above system. Consider $\beta=$ $x-f_{1}^{2}+f_{2}^{2}$ and $\gamma=2 f_{1} f_{2}$, in (9), we obtain

$$
\begin{aligned}
& f_{1}^{\prime \prime} \\
& \quad=\left(x^{2}-f_{1}^{2}+f_{2}^{2}\right)\left(f_{1}^{\prime 3}-3 f_{1}^{\prime} f_{2}^{\prime 2}\right)+2 f_{1} f_{2}\left(3 f_{1}^{\prime 2} f_{2}^{\prime}-f_{2}^{\prime 3}\right) ; \\
& f_{2}^{\prime \prime} \\
& \quad=\left(x^{2}-f_{1}^{2}+f_{2}^{2}\right)\left(3 f_{1}^{\prime 2} f_{2}^{\prime}-f_{2}^{\prime 3}\right)-2 f_{1} f_{2}\left(f_{1}^{\prime 3}-3 f_{1}^{\prime} f_{2}^{\prime 2}\right) .
\end{aligned}
$$

This system has no real point symmetry. However the corresponding complex equation

$$
u^{\prime \prime}-x u^{2} u^{\prime 3}=0
$$

is again linearizable to $U^{\prime \prime}=0$, producing the solution of system (46).

\section{Conclusion}

Complex symmetry analysis provides a class of systems of two ODEs obtainable from a scalar second order equation if the dependent variable is a complex function of a real independent variable. The linearizability of this base complex equation generates two different classes of systems of two second order ODEs: (a) real linearizable systems that can be linearized from real Lie point transformations; and (b) complex linearizable systems that can only be linearized using complex Lie point transformations. In [14], it was shown that systems that can be transformed into a complex linear equation has 6,7 , or 15 maximum dimensional algebra which can, therefore, also be linearized by real Lie point transformations. The second class is investigated here and it is found that these systems are not linearizable by any real Lie point transformations but they are solvable due to their correspondence with linearizable scalar complex equations. This class contains complex linearizable systems with maximum dimensions of Lie algebras less than four. The complex point transformations play a significant role in their linearization.

It would be worthwhile to investigate the contact symmetries of given systems that might provide deeper insights into complex point transformations. The group of contact transformations is infinite-dimensional for linear systems 
TABLE 2: Lie canonical forms of complex scalar equations.

\begin{tabular}{lcc}
\hline Type & Complex symmetry generators & Representative equations \\
\hline I & $Z_{1}=\partial_{x}, Z_{2}=\partial_{u}$, & $u^{\prime \prime}=w\left(u^{\prime}\right)$ \\
II & $Z_{1}=\partial_{u}, Z_{2}=x \partial_{u}$, & $u^{\prime \prime}=w(x)$ \\
III & $Z_{1}=\partial_{u}, Z_{2}=x \partial_{x}+u \partial_{u}$, & $x u^{\prime \prime}=w\left(u^{\prime}\right)$ \\
IV & $Z_{1}=\partial_{u}, Z_{2}=u \partial_{u}$, & $u^{\prime \prime}=u^{\prime} w(x)$
\end{tabular}

and contains the group of fibre-preserving transformations and point transformations as its special cases. The last system with no symmetry clearly indicates an inherited beauty in the complex domain which is not visible in the real domain. It is a benchmark problem as to why this system is solvable in the complex domain and not in the real.

Important features of the given systems can be explored in terms of first integrals by applying the Noether symmetry analysis, provided the given systems come from a variational principle. In contrast, Cartan's equivalence approach (which does not necessarily depend on the existence of a Lagrangian) can be applied to investigate invariants and differential invariants of such systems. Further directions may include the problem of linearization of even dimensional systems of ODEs from complex point transformations.

\section{Appendix}

For completeness we also examine the solvability of those systems that are not complex linearizable yet can be solved via complex procedure if they are mapped to solvable (integrable) scalar complex equations with two-dimensional solvable algebras. For this purpose we state the general form of scalar equations with two symmetries. The integration strategies developed to solve a scalar complex second order ODE (4) require a two parameter complex group (see, e.g., $[1,2]$ ) called $\mathbf{G}_{2}$. The integrable forms of complex second order equations admitting $\mathbf{G}_{2}$ are given in Table 2 .

The following theorem summarizes the complex method to solve systems of ODEs due to their correspondence with the complex scalar solvable second order ODEs.

Theorem A.1. A system of two second order ODEs (3) is solvable regardless of the number of symmetries if the corresponding complex scalar equation (4) is

(i) integrable; that is, it has a two parameter group $\mathbf{G}_{2}$; or

(ii) linearizable via invertible complex point transformations of the form $\mathscr{L}_{2}:(x, u) \rightarrow(\chi, U)$.

Now we give an example of a solvable system which is neither complex linearizable nor linearizable.

Example A.2. Consider a nonlinear coupled system

$$
\begin{aligned}
& f_{1}^{\prime \prime}=\frac{\left(f_{1}^{2}-f_{2}^{2}\right) f_{1}^{\prime}}{\left(f_{1}^{2}-f_{2}^{2}\right)^{2}+4 f_{1}^{2} f_{2}^{2}}+\frac{2 f_{1} f_{2} f_{2}^{\prime}}{\left(f_{1}^{2}-f_{2}^{2}\right)^{2}+4 f_{1}^{2} f_{2}^{2}}, \\
& f_{2}^{\prime \prime}=\frac{\left(f_{1}^{2}-f_{2}^{2}\right) f_{2}^{\prime}}{\left(f_{1}^{2}-f_{2}^{2}\right)^{2}+4 f_{1}^{2} f_{2}^{2}}-\frac{2 f_{1} f_{2} f_{1}^{\prime}}{\left(f_{1}^{2}-f_{2}^{2}\right)^{2}+4 f_{1}^{2} f_{2}^{2}},
\end{aligned}
$$

which has a two-dimensional algebra $\left[X_{1}, X_{2}\right]=2 X_{1}$, where

$$
X_{1}=\frac{\partial}{\partial x}, \quad X_{2}=2 x \frac{\partial}{\partial x}+f_{1} \frac{\partial}{\partial f_{1}}+f_{2} \frac{\partial}{\partial f_{2}} .
$$

Using standard Lie analysis it is not straightforward to carry out integration of this system. Here we highlight the crucial steps involved in using the complex transformations (2) in the form of invariants and differential invariants of symmetries. We first observe that $\omega_{1}$ and $\omega_{2}$ in (3), given by the righthand sides of system (A.1) satisfy CR-equations (5); therefore system (A.1) can be mapped to a scalar complex equation. Indeed, the equation

$$
u^{\prime \prime}=\frac{u^{\prime}}{u^{2}}
$$

corresponds to system (A.1) and it has two complex symmetries $X_{1}$ and $2 x \partial_{x}+u \partial_{u}$; therefore (A.3) has a solvable Lie algebra $\mathbf{G}_{2}$. The integration of above equation can be obtained using both approaches, canonical coordinates or differential invariants. Since scaling is inherited under $X_{1}$ therefore we employ canonical coordinates relative to symmetry $X_{1}$. The canonical transformation

$$
\chi=u, \quad \psi=x, \quad U(\chi)=\frac{d \psi}{d x}=\frac{1}{u^{\prime}},
$$

converts (A.3) into a first-order equation

$$
U^{\prime}=\frac{U^{2}}{\chi^{2}}
$$

which upon realification yields a system of partial differential equations as $\chi$ is a complex independent variable. That is why the system (A.1) is reduced to a pair of first-order partial differential equations rather ODEs. This is a similar situation that arises in complex linearization except the difference that here the target equation is a reduced solvable ODE not a linear equation. By integrating the above equation and using invertible transformation we obtain the solution

$$
\begin{gathered}
2 c_{1} f_{1}+\ln \left(\left(c_{1} f_{1}-1\right)^{2}+c_{2}^{2} f_{2}^{2}\right)-2 c_{1}^{2} x-2 c_{1}^{2} c_{2}=0, \\
c_{1} f_{2}+\arctan \left(\frac{c_{1} f_{1}-1}{c_{1} f_{2}}\right)=0,
\end{gathered}
$$

of system (A.1).

\section{Conflict of Interests}

The authors declare that there is no conflict of interests regarding the publication of this paper.

\section{Acknowledgments}

The authors are grateful to Fazal M. Mahomed (South Africa) for useful comments and discussion on this work. The authors thank the referees for their useful comments which have improved the paper. 


\section{References}

[1] P. J. Olver, Applications of Lie Groups to Differential Equations, vol. 107 of Graduate Texts in Mathematics, Springer, New York, NY, USA, 2nd edition, 1993.

[2] N. H. Ibragimov, Elementary Lie Group Analysis and Ordinary Differential Equations, John Wiley \& Sons, Chichester, UK, 1999.

[3] M. E. Fels, "The equivalence problem for systems of secondorder ordinary differential equations," Proceedings of the London Mathematical Society. Third Series, vol. 71, no. 1, pp. 221-240, 1995.

[4] A. González López, "Symmetries of linear systems of secondorder ordinary differential equations," Journal of Mathematical Physics, vol. 29, no. 5, pp. 1097-1105, 1988.

[5] F. M. Mahomed and A. Qadir, "Invariant linearization criteria for systems of cubically nonlinear second-order ordinary differential equations," Journal of Nonlinear Mathematical Physics, vol. 16, no. 3, pp. 283-298, 2009.

[6] A. V. Aminova and N. A. Aminov, "Projective geometry theory of systems of second-order differential equations: straightening and symmetry theorems," Sbornik Mathematics, vol. 201, pp. 631-643, 2010.

[7] J. Merker, "Characterization of the Newtonian free particle system in $m \geq 2$ dependent variables," Acta Applicandae Mathematicae, vol. 92, no. 2, pp. 125-207, 2006.

[8] Y. Y. Bagderina, "Linearization criteria for a system of two second-order ordinary differential equations," Journal of Physics A: Mathematical and Theoretical, vol. 43, no. 46, Article ID 465201, 14 pages, 2010.

[9] S. V. Meleshko, S. Moyo, and G. F. Oguis, "On the group classification of systems of two linear second-order ordinary differential equations with constant coefficients," Journal of Mathematical Analysis and Applications, vol. 410, no. 1, pp. 341347, 2014.

[10] L. G. Duarte, I. C. Moreira, and F. C. Santos, "Linearization under non-point transformations," Journal of Physics A: Mathematical and General, vol. 27, no. 19, pp. L739-L743, 1994.

[11] V. K. Chandrasekar, M. Senthilvelan, and M. Lakshmanan, "On the complete integrability and linearization of nonlinear ordinary differential equations. V. Linearization of coupled secondorder equations," Proceedings of The Royal Society of London, Series A: Mathematical, Physical and Engineering Sciences, vol. 465, no. 2108, pp. 2369-2389, 2009.

[12] V. K. Chandrasekar, M. Senthilvelan, and M. Lakshmanan, "A systematic method of finding linearizing transformations for nonlinear ordinary differential equations II: extension to coupled ODEs," Journal of Nonlinear Mathematical Physics, vol. 19, no. 2, Article ID 1250013, pp. 203-225, 2012.

[13] C. Wafo Soh and F. M. Mahomed, "Symmetry breaking for a system of two linear second-order ordinary differential equations," Nonlinear Dynamics, vol. 22, no. 1, pp. 121-133, 2000.

[14] M. Safdar, A. Qadir, and S. Ali, "Linearizability of systems of ordinary differential equations obtained by complex symmetry analysis," Mathematical Problems in Engineering, vol. 2011, Article ID 171834, 17 pages, 2011.

[15] S. Ali, F. M. Mahomed, and A. Qadir, "Linearizability criteria for systems of two second-order differential equations by complex methods," Nonlinear Dynamics, vol. 66, no. 1-2, pp. 77-88, 2011. 


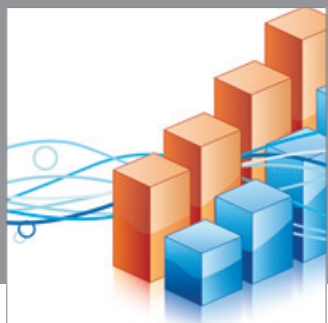

Advances in

Operations Research

mansans

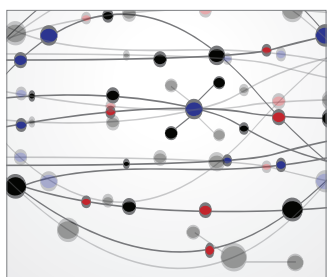

The Scientific World Journal



Submit your manuscripts at http://www.hindawi.com
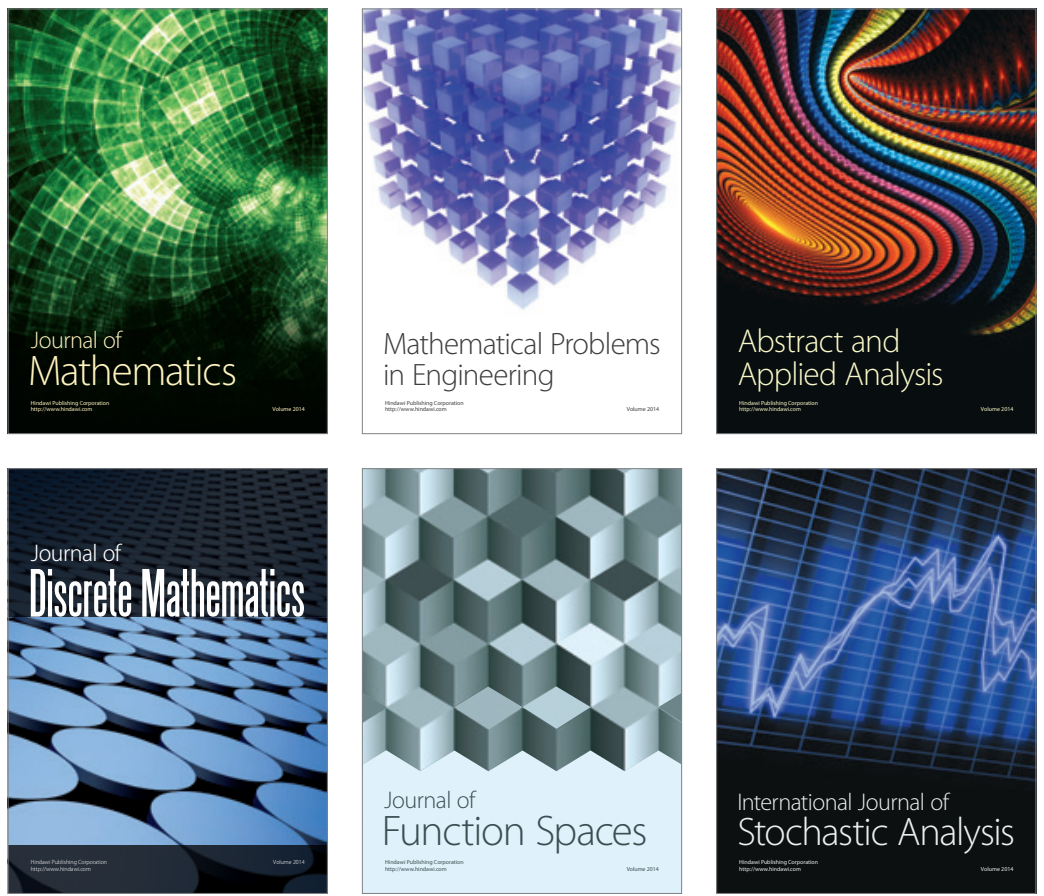

Journal of

Function Spaces

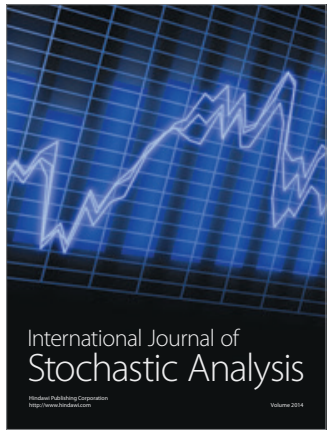

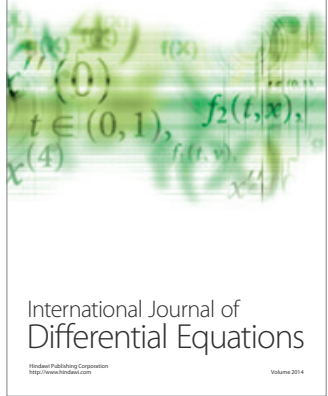
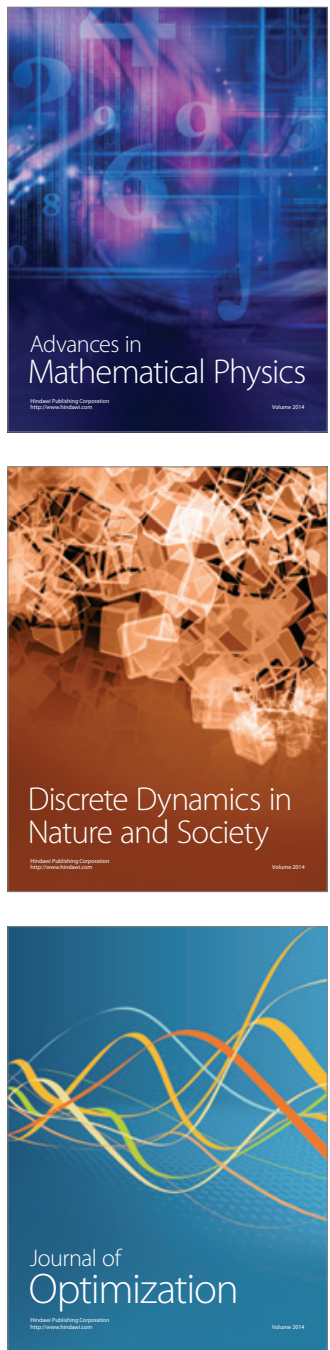